\title{
Future Treatment Option for Hepatocellular Carcinoma: A Focus on Brivanib
}

\author{
Masatoshi Kudo \\ Department of Gastroenterology and Hepatology, Kinki University School of Medicine, Osaka, Japan
}

\section{Key Words}

Hepatocellular carcinoma • Molecular targeted therapy •

Brivanib · Sorafenib

\begin{abstract}
Hepatocellular carcinoma (HCC), one of the most common cancers worldwide, is particularly prevalent in the Asia-Pacific region. Guidelines on the treatment of HCC in Japan come from both consensus-based and evidence-based treatment algorithms. However, patients with extensive liver damage and/or more advanced disease (major vascular invasion and/ or extrahepatic spread) are currently ineligible for any treatment. Recent knowledge of hepatocarcinogenesis has led to the targeting of new pathways, particularly the angiogenic pathway, with a specific focus on the vascular endothelial growth factor receptor (VEGFR). Apparently the most studied systemic antiangiogenic agent for HCC is sorafenib. An updated version of the aforementioned treatment algorithms recommends sorafenib therapy for advanced HCC patients with Child-Pugh A liver function and extrahepatic spread or major vascular invasion. Moreover, sorafenib is recommended for use in HCC patients who are refractory or intolerant to transarterial chemoembolization (TACE) with well-preserved liver function (Child-Pugh A). However, one of the unresolved issues is anti-VEGF resistance. It is speculated that novel antiangiogenic agents that combine inhibition of other pathways such as fibroblast growth factor
\end{abstract}

(C) 2011 S. Karger AG, Basel

0257-2753/11/0293-0316\$38.00/0

Fax +4161306 1234

E-Mail karger@karger.ch

www.karger.com
Accessible online at: www.karger.com/ddi receptor signaling in addition to VEGFR signaling might provide a potential mechanism to overcome anti-VEGF resistance in HCC. Brivanib inhibits both VEGF and fibroblast growth factor receptor signaling. To further investigate the benefits of brivanib for advanced HCC, a broad-spectrum, global, phase III development plan, the Brivanib studies in HCC patients at RISK (BRISK) clinical program, has been initiated. Clinical benefits seen with brivanib in the first-line setting, and following the failure of sorafenib therapy, highlight the potential to improve the clinical course of patients with advanced HCC, and this agent may provide a novel therapeutic option for the growing population of patients for whom no other treatment choice exists.

Copyright $\odot 2011$ S. Karger AG, Basel

\section{Introduction}

Hepatocellular carcinoma (HCC), one of the most common cancers worldwide, is particularly prevalent in the Asia-Pacific region, with more than two thirds of global cases occurring in Asia-Pacific countries [1,2]. In Japan, HCC is now the third leading cause of cancer death among males and females, and is responsible for the death of more than 33,000 Japanese citizens every year [3]. Throughout the Asia-Pacific region, the most important etiologic factors related to HCC are hepatitis B virus (HBV) and hepatitis $\mathrm{C}$ virus (HCV). Among Japanese

Masatoshi Kudo, MD, PhD

Department of Gastroenterology and Hepatology

Kinki University School of Medicine

377-2, Ohno-Higashi, Osaka-Sayama, Osaka 589-8511 (Japan)

Tel. +81 723660221 ext. 3149, E-Mail m-kudo@ med.kindai.ac.jp 
HCC patients, the primary etiology is $\mathrm{HCV}$, with approximately $70-80 \%$ chronically infected with HCV and only a small proportion with $\mathrm{HBV}(<16 \%)[4,5]$.

\section{Treatment Algorithm and Unmet Medical Needs}

Guidance on the treatment of HCC in Japan comes from both consensus-based and evidence-based treatment algorithms [6, 7]. As nationwide HCC screening programs are common in Japan, most patients present in the early stages of the disease and are eligible for potentially 'curative' treatments, such as surgical resection or local ablation (radiofrequency ablation or percutaneous ethanol injection) [6, 7]. If resection or ablation is contraindicated, or if the disease has progressed, then transarterial chemoembolization (TACE) or hepatic infusion chemotherapy may be recommended [6,7]; however, patients with extensive liver damage and/or more advanced disease (major vascular invasion and/or extrahepatic spread) are currently ineligible for these treatments [6]. As such, there remains a significant unmet medical need for patients with advanced HCC in Japan.

\section{Present Status of Molecular Targeted Therapy}

Recent knowledge of hepatocarcinogenesis has led to the targeting of new pathways, particularly the angiogenic pathway, with a specific focus on the vascular endothelial growth factor receptor (VEGFR). Indeed, agents that inhibit angiogenesis via blockade of the VEGFR have seen some success in the treatment of HCC. Moreover, recent research data suggest the potential for an additional synergistic role for antiangiogenic agents whereby they might be used following TACE therapy to increase response rates [8]. Apparently the most studied systemic antiangiogenic agent for HCC is sorafenib. This is an oral multikinase inhibitor that targets the tyrosine kinase activity of VEGFRs 1, 2, and 3, as well as platelet-derived growth factor receptor- $\beta$, and has recently demonstrated some efficacy over placebo in Child-Pugh A patients with advanced HCC [9]. Similar results have been observed in a study of sorafenib for patients with advanced HCC conducted in the Asia-Pacific region [10], and a recent phase 1 study has indicated favorable safety/tolerability and promising antitumor activity in a Japanese population [11]. On the basis of these results, sorafenib has been approved in Japan for the treatment of advanced HCC since May 2009.

Future Treatment Option for HCC: A Focus on Brivanib

\section{Indication of Sorafenib in Treatment Algorithm}

There are, however, unresolved issues regarding the optimal use of sorafenib for HCC. To date, survival benefits in clinical trials have been modest, and a relatively high incidence of hand-foot syndrome (all-grade events reported in $\sim 20-45 \%$ of patients) $[9,10]$ and an increased risk of bleeding events have been reported in the international literature [12]. In Japan, primarily due to the design of the pivotal trials and the available data in HCC patients, sorafenib use has been strictly regulated and limited to patients with Child-Pugh A cirrhosis who are not candidates for resection, ablation, or TACE. Moreover, post-marketing surveillance of sorafenib in Japan has raised safety concerns regarding interstitial pneumonia, hepatic coma, and hepatic failure, which has led to revision of the Japanese package insert. Updated version of the aforementioned treatment algorithms recommend sorafenib therapy for advanced HCC patients with ChildPugh A liver function and extrahepatic spread or major vascular invasion. Moreover, sorafenib is recommended for use in HCC patients who are refractory or intolerant to TACE with well-preserved liver function (Child-Pugh A) (for details, see Kudo, fig. 7, p. 299) [13-15].

\section{Anti-VEGF Resistance}

Recent studies suggest that tumor progression following treatment with antiangiogenic agents that target the VEGF signaling pathway alone may result from either evasive or intrinsic resistance [16]. Furthermore, there is strong evidence to support the hypothesis that evasive resistance to anti-VEGF blockade is associated with reactivation of tumor angiogenesis by alternative signaling pathways, one such mechanism of resistance being activation of the fibroblast growth factor (FGF) signaling pathway $[17,18]$. Basic FGF (FGF2) is a potent angiogenic factor. Indeed, expression of FGF2 enhances growth, invasion, and angiogenesis of many tumor types $[19,20]$. Moreover, recent evidence has shown that FGF is overexpressed and activated in HCC and that high FGF2 levels may predict for a poor clinical outcome among patients with HCC [20].

\section{Importance of FGF Signaling}

Considering the proposed importance of FGF signaling in HCC angiogenesis, it is clear that novel antiangiogenic agents that combine inhibition of FGF receptor sig-

Dig Dis 2011;29:316-320 
Fig. 1. Targeting angiogenesis: mechanisms of resistance. Brivanib may be effective for the failure or resistance of first-line antiangiogenic therapy for VEGF.

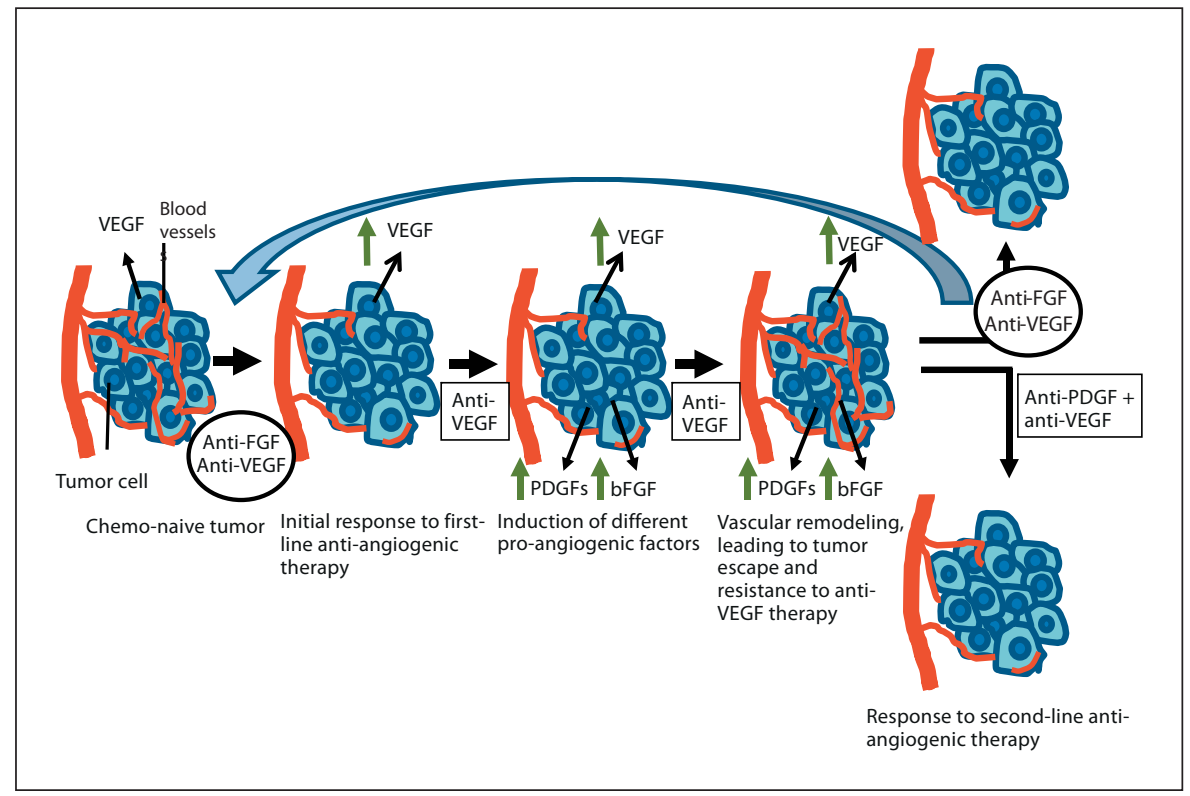

naling with inhibition of VEGFR signaling might provide a potential mechanism to overcome anti-VEGF resistance in HCC (fig. 1). With this in mind, it is worthwhile considering the potential future impact of brivanib on the treatment of advanced HCC. Brivanib, a smallmolecule tyrosine kinase inhibitor, is the first oral selective dual inhibitor of FGF and VEGF signaling. In multiple preclinical models of human xenograft tumors, including patient-derived HCC xenografts, brivanib has shown potent antitumor activity and no overt toxicity when dosed orally [21, 22]. Furthermore, brivanib has demonstrated promising antitumor activity and acceptable tolerability in a phase 2 , open-label study in patients with unresectable locally advanced or metastatic HCC $[23,24]$. Crucially, within this trial, brivanib showed activity both as first-line therapy (overall survival: 10 months) or as second-line therapy in patients who had failed prior antiangiogenic treatment, primarily with sorafenib (overall survival 9.5 months) [24]. Of note, the incidence of all-grade hand-foot syndrome was only $8 \%$ in this study.

\section{Phase I and II Data of Brivanib}

Additional retrospective studies and subanalyses have also confirmed that brivanib is effective in patients from the Asia-Pacific region. In a subanalysis performed to evaluate the effects of brivanib among Asian versus non-
Asian patients enrolled in the aforementioned phase II study, median overall survival was 10.6 months among Asian patients treated with first-line brivanib (versus 5.7 months in non-Asian patients) and 9.8 months among Asian patients receiving brivanib as second-line therapy (versus 9.4 months in non-Asian patients) [25]. Another subanalysis, this time including only patients who received first-line brivanib therapy in the phase 2 study, indicated that overall tolerability was similar or slightly better in the Asian population versus non-Asian patients [26]. A further subanalysis comparing 125 Asian and non-Asian patients enrolled in separate phase I and II studies $[23,27]$ confirmed that exposures in these patient subpopulations were similar following brivanib doses of $800 \mathrm{mg}$ daily [28]. Finally, a phase 1 study of brivanib in Japanese patients with advanced or metastatic solid tumors, including HCC, has shown manageable tolerability and a similar safety profile at the same 800 -mg once-daily dose as used in Caucasian patients [29]. Moreover, the study provided evidence of antitumor activity in this uniquely Japanese population, with 8 of 13 patients (62\%) showing stable disease.

\section{Design of Phase III Global Study}

To further investigate the benefits of brivanib for advanced HCC, a broad-spectrum, global, phase III development plan, the Brivanib studies in HCC patients at 
RISK (BRISK) clinical program, has been initiated. The global BRISK program will enroll patients from countries in Africa, Asia (including Japan), Australasia, Europe, and North, South, and Central America, and will include investigations of brivanib in a variety of clinically relevant settings, including first-line head to head with sorafenib, second-line post-sorafenib, and TACE adjuvant settings. In addition, it is noteworthy that the BRISK study of brivanib as adjuvant treatment to TACE therapy is being led by Japanese investigators and is one of the first global registration programs to be led from Japan.

\section{Conclusion}

HCC continues to be a major healthcare burden in Japan. Although it is detected in the early stages in most Japanese patients and treated accordingly, there remains a population of patients with advanced HCC who have limited therapeutic choices. With the recent approval of the antiangiogenic agent sorafenib, options for these patients have improved, but clinical studies to date suggest only a modest survival benefit with sorafenib and there is potential for safety/tolerability issues and the development of resistance to the anti-VEGF blockade. Clinical benefits seen with brivanib in the first-line setting, and following the failure of sorafenib therapy, highlight the potential to improve the clinical course of patients with advanced HCC, and this agent may provide a novel therapeutic option for the growing population of patients for whom no other treatment choice exists.

\section{Disclosure Statement}

The author has no conflict of interest to declare.

\section{References}

1 Parkin DM, Bray F, Ferlay J, Pisani P: Global cancer statistics, 2002. CA Cancer J Clin 2005;55:74-108.

$>2$ El Serag HB, Rudolph KL: Hepatocellular carcinoma: epidemiology and molecular carcinogenesis. Gastroenterology 2007;132: 2557-2576.

3 Cancer Statistics in Japan 2009. Tokyo, Foundation for Promotion of Cancer Research, 2009.

-4 Takayasu K, Choi BI, Wang CK, et al: First international symposium of current issues for nationwide survey of primary liver cancer in Korea, Taiwan and Japan. Jpn J Clin Oncol 2007;37:233-240.

5 Yuen MF, Hou JL, Chutaputti A: Hepatocellular carcinoma in the Asia-Pacific region. J Gastroenterol Hepatol 2009;24:346-353.

$\checkmark 6$ Kudo M, Okanoue T: Management of hepatocellular carcinoma in Japan: consensusbased clinical practice manual proposed by the Japan Society of Hepatology. Oncology 2007;72(suppl 1):2-15.

7 Makuuchi M, Kokudo N, Arii S, Igaki H, Ikai I, Kaneko S, Kawasaki S, Kudo M, Matsuyama Y, Ohtomo K, et al: Surveillance algorithm and diagnostic algorithm for hepatocellular carcinoma. Hepatol Res 2010;40 (suppl 1):1-144.

8 Xiao EH, Guo D, Bian DJ: Effect of preoperative transcatheter arterial chemoembolization on angiogenesis of hepatocellular carcinoma cells. World J Gastroenterol 2009;15: 4582-4586.
$>9$ Llovet JM, Ricci S, Mazzaferro V, et al: Sorafenib in advanced hepatocellular carcinoma. N Engl J Med 2008;359:378-390.

10 Cheng AL, Kang YK, Chen Z, et al: Efficacy and safety of sorafenib in patients in the Asia-Pacific region with advanced hepatocellular carcinoma: a phase III randomised, double-blind, placebo-controlled trial. Lancet Oncol 2009; 10:25-34.

-11 Furuse J, Ishii H, Nakachi K, Suzuki E, Shimizu S, Nakajima K: Phase I study of sorafenib in Japanese patients with hepatocellular carcinoma. Cancer Sci 2008;99:159165.

12 Je Y, Schutz FA, Choueiri TK: Risk of bleeding with vascular endothelial growth factor receptor tyrosine-kinase inhibitors sunitinib and sorafenib: a systematic review and meta-analysis of clinical trials. Lancet Oncol 2009; 10:967-974.

13 Kudo M: The 2008 Okuda Lecture. Management of hepatocellular carcinoma: from surveillance to molecular targeted therapy. J Gastroenterol Hepatol 2010;25:439-452.

14 Arii S, Sata M, Sakamoto M, Shimada M, Kumada T, Shiina S, Yamashita T, Kokudo N, Tanaka M, Takayama T, et al: Management of hepatocellular carcinoma. Report of Consensus Meeting in the 45th Annual Meeting of the Japan Society of Hepatology, 2009. Hepatol Res 2010;40:667-685.
15 Kudo M, Izumi N, Kokudo N, Matsui O, Sakamoto M, Nakashima O, Kojiro M, Makuuchi M; for the HCC Expert Panel of Japan Society of Hepatology: Management of hepatocellular carcinoma in Japan: consensus-based clinical practice guidelines proposed by the Japan Society of Hepatology (JSH) 2010 updated version. Dig Dis 2011; 29:339-364.

16 Bergers G, Hanahan D: Modes of resistance to anti-angiogenic therapy. Nat Rev Cancer 2008;8:592-603.

17 Batchelor TT, Sorensen AG, di Tomaso E, et al: AZD2171, a pan-VEGF receptor tyrosine kinase inhibitor, normalizes tumor vasculature and alleviates edema in glioblastoma patients. Cancer Cell 2007;11:83-95.

18 Kopetz S, Hoff PM, Eng MJ, et al: Angiogenic cytokines are increased prior to disease progression in metastatic colorectal cancer patients treated with bevacizumab. ASCO Gastrointestinal Cancers Symposium, San Francisco, January 15-17, 2009, abstr 292.

19 El Assal ON, Yamanoi A, Ono T, Kohno H, Nagasue N: The clinicopathological significance of heparanase and basic fibroblast growth factor expressions in hepatocellular carcinoma. Clin Cancer Res 2001;7:12991305.

20 Poon RT, Ng IO, Lau C, Yu WC, Fan ST, Wong J: Correlation of serum basic fibroblast growth factor levels with clinicopathologic features and postoperative recurrence in hepatocellular carcinoma. Am J Surg 2001;182:298-304. 
21 Huynh H, Ngo VC, Fargnoli J, et al: Brivanib alaninate, a dual inhibitor of vascular endothelial growth factor receptor and fibroblast growth factor receptor tyrosine kinases, induces growth inhibition in mouse models of human hepatocellular carcinoma. Clin Cancer Res 2008;14:6146-6153.

22 Bhide RS, Lombardo LJ, Hunt JT, et al: The antiangiogenic activity in xenograft models of brivanib alaninate, a dual inhibitor of VEGFR-2 and FGFR-1 kinases. Mol Cancer Ther 2010;9:369-378.

23 Raoul JL, Finn R, Kang YK, et al: An openlabel phase II study of first- and second-line treatment with brivanib in patients with hepatocellular carcinoma. ASCO General Meeting, Orlando, May 29-June 2, 2009, abstr 4577.
24 Raoul JL, Finn R, Kang YK, et al: Phase 2 study of first- and second-line treatment with brivanib in patients with hepatocellular carcinoma. ILCA Third Annual Conference, Milan, September 4-6, 2009, abstr O-030.

25 Chao Y, Kang YK, Park JW, et al: Phase 2 study of first- and second-line treatment with brivanib in patients with hepatocellular carcinoma (HCC). 12th Chinese Society for Clinical Oncology Annual Meeting, Xiamen, China, October 14-18, 2009.

26 Park JW, Harris R, Barrett B, Baudelet C, Walters I: A subanalysis of Asian and nonAsian patients with hepatocellular carcinoma treated in a phase 2 safety and efficacy study of brivanib as first-line therapy. Asian Pacific Association for the Study of the Liver (APASL), Hong Kong, April 13-16, 2009, abstr PE255.
27 Garrett C, Siu L, El-Khoueiry A, et al: A phase I study of brivanib alaninate (BMS582664), an oral dual inhibitor of VEGFR and FGFR tyrosine kinases, in combination with full-dose cetuximab in patients with advanced gastrointestinal malignancies who failed prior therapy. J Clin Oncol 2008;26 (suppl):abstr 4111.

28 Wang X, Syed S, Masson E, Walters I, Amit A, Zhang L: Characterization of brivanib pharmacokinetics in Asian and non-Asian subjects with advanced or metastatic solid tumors. Asian Pacific Association for the Study of the Liver (APASL), Hong Kong, April 13-16, 2009, abstr PE256.

29 Hironaka S, Onozawa Y, Aramaki T, et al: Phase 1 study of brivanib alaninate, a novel FGF and VEGF receptor inhibitor. Japanese Society for Clinical Oncology (JSCO) Annual Conference, Yokohama, October 2224, 2009, abstr OS036-3. 